\title{
Redesign de aplicativos para pessoas com Transtorno do Espectro Autista orientado pelas diretrizes do GAIA
}

\author{
Bruno de Mendonça \\ Universidade Federal \\ do Ceará \\ Russas, CE, Brasil \\ brunohdem@alu.ufc.br \\ Carlos J. Silva Lopes \\ Universidade Federal \\ do Ceará \\ Russas, CE, Brasil \\ carlosjardel@alu.ufc.br
}

\author{
Marília S. Mendes \\ Universidade Federal \\ do Ceará \\ Russas, CE, Brasil \\ marilia.mendes@ufc.br
}

\author{
Anna Beatriz Marques \\ Universidade Federal do \\ Ceará \\ Russas, CE, Brasil \\ beatriz.marques@ufc.br
}

\begin{abstract}
RESUMO
Pessoas com o Transtorno do Espectro Autista (TEA) apresentam dificuldades na interação social, comunicação e um repertório restrito de interesses e atividades. Devido às dificuldades para entender expressões, as pessoas com TEA podem não compreender ou perceber determinados conteúdos, termos e imagens presentes na interface de aplicações. Pesquisas recentes propõem diretrizes para a construção de interfaces adequadas a esses usuários, como o GAIA (Guia de Acessibilidade de Interfaces Web com foco em aspectos do Autismo). Porém, ainda não existem relatos sobre o uso dessas diretrizes no redesign de interface de aplicações. Esse artigo apresenta a adoção das diretrizes do GAIA para o redesign de interface de dois aplicativos móveis para pessoas com TEA.
\end{abstract}

\section{Palavras-chave}

Transtorno do Espectro Autista; design de interface; acessibilidade; diretrizes.

\section{ACM Classification Keywords}

Human-centered computing $\rightarrow$ Accessibility $\rightarrow$ Accessibility design and evaluation methods.

\section{INTRODUÇÃO E CARACTERIZAÇÃO DO PROBLEMA}

O Transtorno do Espectro Autista (TEA) é caracterizado por prejudicar as habilidades de interação social, comunicação dos indivíduos, além da presença de comportamentos repetitivos e estereotipados [1]. Devido às dificuldades para entender expressões, usuários com TEA podem não compreender ou perceber determinados conteúdos, termos e figuras presentes na interface de aplicações. Por isso, pesquisas recentes propõem diretrizes para a construção de interfaces acessíveis a autistas, como o projeto GAIA (Guia de Acessibilidade de Interfaces Web com foco em aspectos do Autismo) [2].

Permission to make digital or hard copies of all or part of this work for personal or classroom use is granted without fee provided that copies are not made or distributed for profit or commercial advantage and that copies bear this notice and the full citation on the first page. Copyrights for components of this work owned by others than the author(s) must be honored. Abstracting with credit is permitted. To copy otherwise, or republish, to post on servers or to redistribute to lists, requires prior specific permission and/or a fee. Copyright 2018 SBC.

IHC 2018, Anais Estendidos do XVII Simpósio Brasileiro sobre Fatores

Humanos em Sistemas Computacionais

Outubro 22-26, 2018, Belém, Brasil

PÔSTERES VIRTUAIS E DEMOS
O GAIA possui 28 diretrizes organizadas em dez grupos: (1) vocabulário visual e textual, (2) customização, (3) engajamento, (4) representações redundantes, (5) multimídia, (6) respostas às ações, (7) affordance, (8) navegabilidade, (9) visibilidade do estado do sistema e (10) interação com telas sensíveis ao toque [2]. Devido à proposta das diretrizes ser recente, ainda não existe um relato do seu uso no redesign de interface. Embora o GAIA não seja específico para aplicativos móveis, o número de tecnologias para dispositivos móveis e direcionadas a pessoas com TEA [3] motivou a condução desta pesquisa.

Essa pesquisa faz parte de um projeto de extensão que visa desenvolver tecnologias acessíveis para o município de Russas (CE). Por meio de pesquisas conduzidas no município, identificou-se que havia em média 50 alunos autistas matriculados nas escolas. A equipe do projeto decidiu oferecer um curso de extensão de desenvolvimento de aplicativos acessíveis com a ferramenta AppInventor a alunos do ensino médio. Os alunos desenvolveram três aplicativos direcionados a pessoas autistas. Dois aplicativos foram avaliados com o uso das diretrizes do GAIA. As necessidades de melhorias identificadas possibilitaram o redesign de sua interface.

\section{METODOLOGIA DE PESQUISA}

A metodologia adotada possui 5 etapas: (1) pesquisa sobre diretrizes de acessibilidade direcionadas a pessoas com TEA; (2) avaliação dos aplicativos com o uso das diretrizes; (3) validação dos resultados da avaliação com especialistas em IHC; (4) redesign da interface dos aplicativos com base nos problemas identificados; (5) validação do redesign da interface com especialistas em IHC. O GAIA [2] foi selecionado por ser baseado em revisão sistemática sobre diretrizes existentes. Em seguida, dois alunos avaliaram os dois aplicativos móveis orientados pelo GAIA, analisando quais diretrizes eram atendidas ou não. $\mathrm{O}$ resultado da avaliação foi apresentado à equipe do projeto, coordenado por dois especialistas em IHC. Então, os dois alunos reprojetaram a interface dos aplicativos de acordo com os problemas encontrados na avaliação. O redesign de interface também foi validado junto à equipe do projeto.

\section{RESULTADOS PRELIMINARES}

$O$ redesign de interface dos aplicativos será resumidamente apresentado, indicando as diretrizes do GAIA adotadas. 


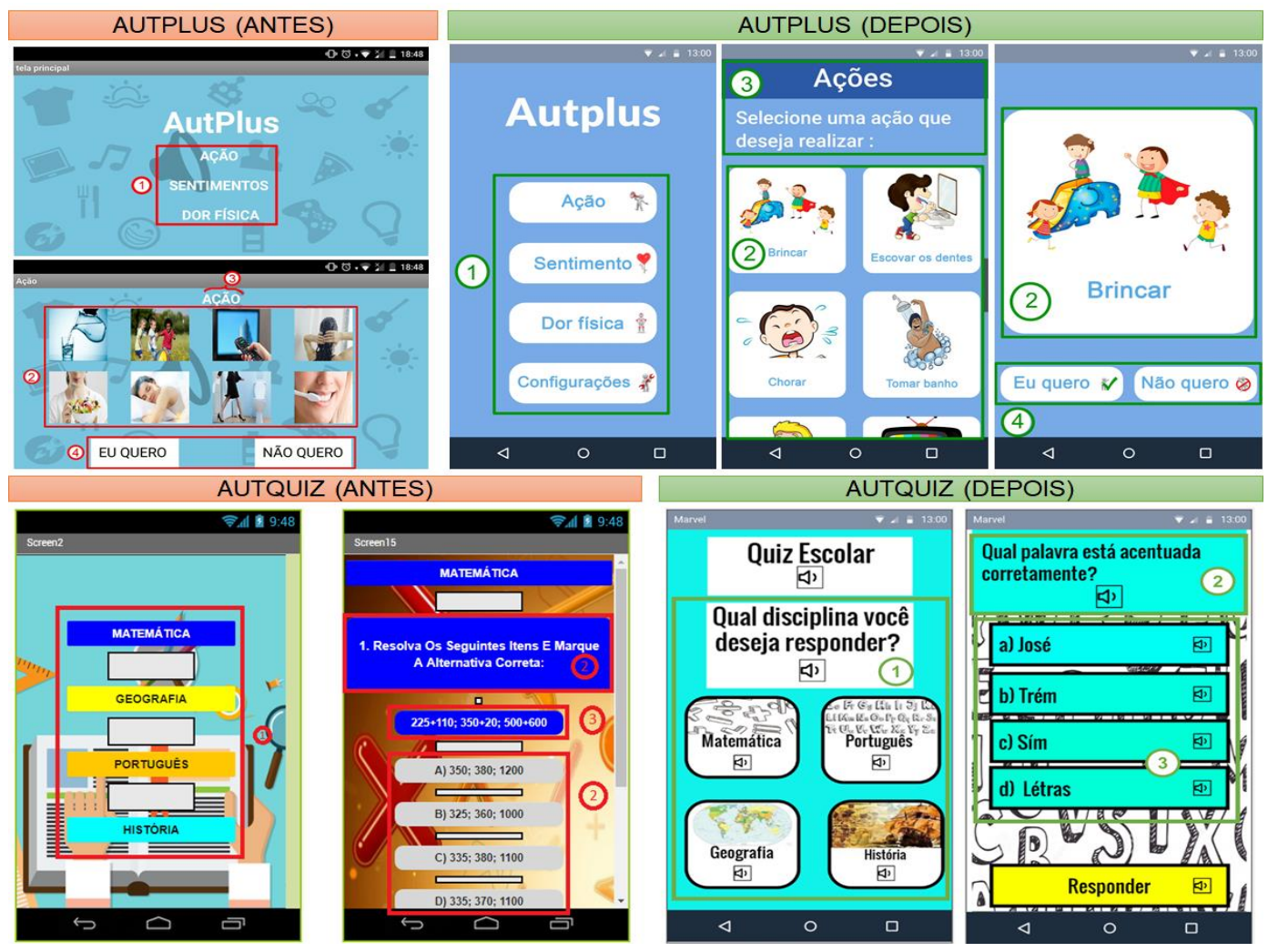

Figura 1. Redesign de interface dos aplicativos AutPlus e AutQuiz.

A Figura 1 exemplifica as melhorias realizadas nos aplicativos por áreas, de (1) a (4). Os novos protótipos foram criados na plataforma Marvel ${ }^{1}$. O AutPlus objetiva auxiliar a comunicação, para que os usuários possam expressar seus sentimentos, dores físicas e necessidades. O AutQuiz busca auxiliar na memorização de conteúdos escolares por meio de um quiz. Na área (1) de ambos os aplicativos, as seguintes diretrizes foram adotadas: [1.1] as cores, contrastes e objetos de primeiro plano devem ser adequados; [2.1] e [2.2] a opção de customização ajuda usuário; [3.3] é importante sempre usar espaçamentos em branco entre elementos; [7.2] ícones e botões devem ter uma área de clique/toque adequada; [5.1] forneça informações em diferentes representações como texto, áudio e imagens para melhor compreensão do conteúdo. Na área (2) do Autplus, além das diretrizes adotadas na área (1), foram aplicadas: [4.1] não devem ser usados somente textos para a apresentação de um conteúdo; [4.2] símbolos, pictogramas e ícones devem apresentar um equivalente textual para facilitar a sua compreensão; [5.2] imagens possam ser ampliadas para melhor visualização. $\mathrm{Na}$ área (3) do AutPlus, foram aplicadas as diretrizes: [1.2] a utilização de uma linguagem visual e textual simples ajuda no uso; [3.4] é importante fornecer instruções sobre as funções ao usuário. $\mathrm{Na}$ área (4) do AutPlus também foi

\footnotetext{
${ }^{1}$ Protótipos do AutPlus: https://marvelapp.com/8ib94g7 e AutQuiz: https://marvelapp.com/3gcfid4
}

adotada a diretriz [1.4]. No AutQuiz, foi adotada a diretriz [4.1] na área (2) e as diretrizes [3.3] e [5.1] na área (3).

\section{CONSIDERAÇÕES FINAIS E TRABALHOS FUTUROS}

Esse trabalho relatou o redesign de aplicativos para usuários com TEA com o uso de diretrizes do GAIA. Espera-se motivar profissionais e pesquisadores de IHC a adotar diretrizes como o GAIA em seus projetos. Como trabalhos futuros, pretende-se avaliar os aplicativos reprojetados por meio de testes com usuários e investigar o uso das diretrizes para avaliar outras aplicações.

\section{REFERÊNCIAS}

1. American Psychiatric Association. 2013. Diagnostic and Statistical Manual of Mental Disorders. American Psychiatric Press.

2. Talita C.G. Britto e Ednaldo B. Pizzolato. 2018. GAIA: uma proposta de um guia de ecomendações de acessibilidade de interfaces Web com foco em aspectos do Autismo. Revista Brasileira de Informática na Educação (RBIE) 26, 2: 102-123.

3. Heloise C. Magaton e Silvia A. Bim. 2017. The Use of Educational Applications by Children with Autistic Spectrum Disorder: A Case of Study. In Proc. of the XVI Brazilian Symposium on Human Factors in Computing Systems (IHC 2017). Article 50, 10 pages. 